\title{
THE JACOBIAN CONJECTURE: SURVEY OF SOME RESULTS
}

\author{
LUDWIK M. DRUŻKOWSKI \\ Institute of Mathematics, Jagiellonian University \\ Reymonta 4/508, 30-059 Kraków, Poland \\ E-mail:druzkows@im.uj.edu.pl
}

\begin{abstract}
The paper contains the formulation of the problem and an almost up-to-date survey of some results in the area.

1. Formulation and some history of the problem. Let $\mathbb{K}$ denote either $\mathbb{C}$ or $\mathbb{R}, F_{j} \in \mathbb{K}\left[X_{1}, \ldots, X_{n}\right], j=1, \ldots, n$ and let

$\mathcal{P}\left(\mathbb{K}^{n}\right)=\left\{F=\left(F_{1}, \ldots, F_{n}\right): \mathbb{K}^{n} \rightarrow \mathbb{K}^{n} ; F_{j} \in \mathbb{K}\left[X_{1}, \ldots, X_{n}\right], j=1, \ldots, n\right\}$, i.e. $\mathcal{P}\left(\mathbb{K}^{n}\right)$ is the set of polynomial mappings of $\mathbb{K}^{n}$. Further let $\operatorname{Jac} F(x):=$ $\operatorname{det}\left[\frac{\partial F_{i}}{\partial x_{j}}(x): i, j=1, \ldots, n\right]$ denote the jacobian of the map $F$ at a point $x=$ $\left(x_{1}, \ldots, x_{n}\right) \in \mathbb{K}^{n}$. Fix $n>1$ and recall the formulation of the $n$-dimensional Jacobian Conjecture (for short $(\mathrm{JC})_{n}$ )

$(\mathrm{JC})_{n} \quad\left[F \in \mathcal{P}\left(\mathbb{K}^{n}\right)\right.$ and $\operatorname{Jac} F(x) \neq 0$ for every $\left.x \in \mathbb{K}^{n}\right] \Rightarrow[F$ is injective $]$, and the so called Generalized Jacobian Conjecture (for short: (GJC)), namely

$(\mathrm{JC})_{n}$ holds for every $n>1$.

If $\mathbb{K}=\mathbb{C}$, then we call the Jacobian Conjecture the complex Jacobian Conjecture (resp. the real Jacobian Conjecture if $\mathbb{K}=\mathbb{R}$ ). Although it is common to call the $n$ dimensional Jacobian Conjecture or Generalized Jacobian Conjecture briefly: the Jacobian Conjecture, to avoid any confusion we use the introduced terminology in the paper. Since $F \in \mathcal{P}\left(\mathbb{C}^{n}\right)$ can be treated as $\widehat{F} \in \mathcal{P}\left(\mathbb{R}^{2 n}\right)$ and Jac $\widehat{F}(x, y)=$ $|\operatorname{Jac} F(x+\mathbf{i} y)|^{2}$, it is evident that

$$
\text { the real (GJC) } \Rightarrow \text { the complex (GJC). }
$$

1991 Mathematics Subject Classification: 14E09, 32H02.

Partially supported by KBN Grant 210779101.

The paper is in final form and no version of it will be published elsewhere.
\end{abstract}


But we do not know if

$$
\text { real }(\mathrm{JC})_{n} \Rightarrow \text { complex }(\mathrm{JC})_{n}
$$

(note that there is a gap in the proof of ? given in [BCW]). If $\mathbb{K}$ is an algebraically closed field with characteristic equal to 0 , then many other equivalent formulations of the complex Jacobian Conjecture are known, cf. e.g. [BCW, D4, Es1-3, KS, R2, St]. Due to Lefschetz Principle it is sufficient in this case to deal only with the complex Jacobian Conjecture if Jac $F=$ const. If $\mathbb{K}=\mathbb{R}$, then equivalent formulations of the real Jacobian Conjecture are also given, cf. e.g. [MO1].

A special case of the complex two dimensional Jacobian Conjecture $(\mathrm{JC})_{2}$, was considered by O.-H. Keller in 1939, cf. [K]. Namely, Keller asked if a polynomial map $F: \mathbb{C}^{2} \rightarrow \mathbb{C}^{2}$, Jac $F=1$, with integer coefficients is a polynomial automorphism, i.e. the inverse $F^{-1}$ exists and is a polynomial mapping with integer coefficients. Hence, the two dimensional complex case of the Jacobian Conjecture is often called Keller's question or Keller's Jacobian Conjecture. Note that if the two dimensional complex Jacobian Conjecture is true, then Keller's Problem has the affirmative answer, cf. e.g. [D4].

Several alleged proofs of the Jacobian Conjecture have been published. The most well-known is Engel's proof, cf. [E], given in 1955. In 1973 A.G. Vitushkin, cf. [V], pointed out two essential errors which invalidated Engel's proof. Between 1956 and 1960 B. Segre published at least three wrong proofs of the Jacobian Conjecture, cf. [S1, S2, S3]. Since his "proofs" were of geometrical character, Segre asked in [S3] for a purely algebraic proof of the Conjecture. In 1961 W. Gröbner, cf. [G], tried to give a purely algebraic proof of the Jacobian Conjecture, but O. Zariski found a computational error which ruined Gröbner's argument and pointed it out to the "MR" reviewer of Gröbner's article. In 1980 S. Oda published, cf. [O], an alleged proof which contains an evidently false lemma. For a nice survey paper concerning, among others, some history of the Jacobian Conjecture we refer the reader to [BCW, D4, Es2, M]. Up to now the Jacobian Conjecture remains unsolved in the real and complex case even if $n=2$.

On the other hand the Jacobian Conjecture is one of questions on the so called Global Injectivity Problem: Let $U$ be an open connected subset of $\mathbb{K}^{n}$, $F \in \mathcal{C}^{1}\left(U, \mathbb{K}^{n}\right)$ and let $\operatorname{Jac} F(x) \neq 0$ for $x \in U$.

? For which classes of mappings the mapping $F$ (defined above) is injective?

The answer to the question is evidently positive in the case of $\mathbb{K}$-linear maps and also if $\mathbb{K}^{n}=\mathbb{R}$. Obviously in the complex case the answer is negative even if $n=1$ and $F$ is holomorphic, e.g. $f(z)=e^{z}, z \in \mathbb{C}$. This example also works for real analytic maps of two variables, namely the map $g(x, y):=\left(\operatorname{Re} e^{z}, \operatorname{Im} e^{z}\right)=$ $\left(e^{x} \cos y, e^{x} \sin y\right)$ has everywhere positive jacobian, but it is not injective. In complex multidimensional case the answer is also negative for holomorphic mappings with nonzero constant jacobian, e.g. $f(z, w)=\left(z e^{-w}, e^{w}\right),(z, w) \in \mathbb{C}^{2}$. Therefore, it is evident that the natural classes of mappings to which we should restrict 
our attention are polynomial and rational maps of $\mathbb{K}^{n}$. The question has the negative answer for complex rational maps, e.g. the map $f(x, y):=\left(x^{2}, \frac{y}{2 x}\right)$, $(x, y) \in U:=\mathbb{C}^{2} \backslash\{x=0\}$ has $\operatorname{Jac} f=1$, but $f$ is not injective on $U$. Thus, in the complex case we have to consider only polynomial maps with constant nonzero jacobians. The problem for rational maps defined everywhere on $\mathbb{R}^{n}$ is not decided yet. However, the following example (cf. [M]) shows difficulties of the problem.

ExAmple 1.1. Identify $(x, y) \in \mathbb{R}^{2}$ with $z=x+\mathbf{i} y \in \mathbb{C}$ and define the mapping $F: \mathbb{R}^{2} \rightarrow \mathbb{R}^{2}$ by the formula

$$
F(x, y):= \begin{cases}\left(\frac{-\operatorname{Re} z^{5}}{|z|^{4}}, \frac{-\operatorname{Im} z^{5}}{|z|^{4}}\right), & \text { when } z \neq 0 \\ (0,0), & \text { when } z=0 .\end{cases}
$$

The mapping $F$ is continuous everywhere on $\mathbb{R}^{2}$, smooth except for the point $z=0$ and $\operatorname{Jac} F(x, y)=\left|\frac{\partial f}{\partial z}\right|^{2}-\left|\frac{\partial f}{\partial \bar{z}}\right|^{2}=5$ when $z=x+\mathbf{i} y=(x, y) \neq(0,0)$,

$$
\operatorname{Jac} f(0,0):=\frac{\partial f_{1}}{\partial x}(0,0) \frac{\partial f_{2}}{\partial y}(0,0)-\frac{\partial f_{1}}{\partial y}(0,0) \frac{\partial f_{2}}{\partial x}(0,0)=1,
$$

but $F$ is not injective since $f(1,0)=(-1,0)=f\left(\cos \frac{2 \pi}{5}, \sin \frac{2 \pi}{5}\right)$.

The following remark is obvious.

Rem ark 1.2. Without loss of generality we can consider in (GJC) (or in $(\mathrm{JC})_{n}$ ) only polynomial mappings having the form $F(X)=X+R(X)$, where $R \in \mathcal{P}\left(\mathbb{K}^{n}\right)$ and ord $R>1$. Then the assumption that Jac $F \neq 0$ everywhere says that $\operatorname{Jac} F=1$, if $\mathbb{K}=\mathbb{C}$ and $\operatorname{Jac} F>0$, if $\mathbb{K}=\mathbb{R}$.

We finish this section with an equivalent formulation of the Jacobian Conjecture.

Proposition 1.3. Let $F \in \mathcal{P}\left(\mathbb{C}^{n}\right)$ and Jac $F=1$. Then

$[F$ is injective $] \Leftrightarrow\left[\Delta(F):=\left\{(x, y) \in \mathbb{C}^{n} \times \mathbb{C}^{n}: F(x)=F(y)\right\}\right.$ is connected $]$.

Pr o of. Assume that $\Delta(F)$ is connected (in Zariski or in euclidean topology of $\left.\mathbb{C}^{n}\right)$. Then $\Delta(F)$ is a smooth algebraic manifold. If $\Delta(F)$ is connected, then, due to the classical theorems, $\Delta(F)$ is a smooth irreducible algebraic set. Evidently the diagonal $D=\left\{(x, y) \in \mathbb{C}^{n} \times \mathbb{C}^{n}: x=y\right\} \subset \Delta(F)$ and $\operatorname{dim} D=\operatorname{dim} \Delta(F)=n$. Since $\Delta(F)$ is irreducible, we get the equality: $\Delta(F)=D$, i.e. $F$ is injective. The converse implication is obvious.

2. Basic facts on polynomial maps. Fortunately polynomial mappings have nice properties not enjoyed by holomorphic mappings, and we recall such properties.

ThEOREM 2.1 ([BR]). Every injective polynomial map of $\mathbb{K}^{n}$ is bijective. 
Note that such a theorem is not true for analytic maps, even in the complex case. If $n>1$, then there exist injective holomorphic maps of $\mathbb{C}^{n}$ with nonzero constant jacobian whose images are not dense in $\mathbb{C}^{n}$ (the Poincaré-FatouBieberbach phenomenon).

TheOREM 2.2 ([BCW, R2, W]. Any injective polynomial map $F$ of $\mathbb{C}^{n}$ is a polynomial automorphism, i.e. the inverse $F^{-1}$ exists and is a polynomial mapping.

Remember that the above theorem is not true in the real case even if $n=1$ and the jacobian of a polynomial mapping $F$ is everywhere different from zero, e.g. $F(x)=x+x^{3}, x \in \mathbb{R}$. If $F$ is a polynomial automorphism, then it is possible to give a sharp estimate for the degree of its inverse, namely

THEOREM 2.3 ([BCW, RW]). If $F$ is a polynomial automorphism of $\mathbb{K}^{n}$, then

$$
\operatorname{deg} F^{-1} \leq(\operatorname{deg} F)^{n-1} \text {. }
$$

For a refined version of the above theorem see $[\mathrm{P}]$. Finaly we recall a theorem about the number of points in the fibre of a polynomial mapping with the jacobian different from zero everywhere.

TheOREM 2.4. Let $F=\left(F_{1}, \ldots, F_{n}\right): \mathbb{K}^{n} \rightarrow \mathbb{K}^{n}$ be a polynomial map such that Jac $F(x) \neq 0$ for every $x \in \mathbb{K}^{n}$.

(i) Then for every $b \in \mathbb{K}^{n}$ the equation $F(x)=b$ has only isolated solutions and

$$
\#\left\{x \in \mathbb{K}^{n}: F(x)=b\right\} \leq \operatorname{deg} F_{1} \cdot \ldots \cdot \operatorname{deg} F_{n} .
$$

(ii) If $\mathbb{K}=\mathbb{C}$, then $\left\{y \in \mathbb{C}^{n}: \# F^{-1}(y)<\max \left\{\# F(b): b \in \mathbb{C}^{n}\right\}\right\}$ is either empty or is an algebraic hypersurface of $\mathbb{C}^{n}$.

(iii) If $\mathbb{K}=\mathbb{R}$, then $\left\{y \in \mathbb{R}^{n}: \# F(y):=\max \left\{\# F(b): b \in \mathbb{R}^{n}\right\}\right\}$ is a nonempty open subset of $\mathbb{R}^{n}$.

Pr o of. If $\mathbb{K}=\mathbb{C}$, then the inequality (i) is the well known Bézout Inequality. If $\mathbb{K}=\mathbb{R}$, then the proof of (i) and (iii) can be found e.g. in [DT, Lemma 3.1]. The proof of (ii) is given in [D4].

3. Elementary algebraic approach: reduction of the degree. We recall the theorem which shows that it is sufficient to consider in the Jacobian Conjecture only polynomial mappings of a special form.

TheOREM 3.1 ([Y, BCW, D1]). If we consider (GJC) (i.e. the Jacobian Conjecture for every $n>1$ ), then it is sufficient to consider, for every $n>1$, only polynomial mappings of the so called cubic homogeneous form $F=I+H$, where $I$ denotes identity, $H=\left(H_{1}, \ldots, H_{n}\right)$ and $H_{j}: \mathbb{K}^{n} \rightarrow \mathbb{K}$ is a cubic homogeneous polynomial of degree 3 or zero, $j=1, \ldots, n$. 
Remark 3.2. The cubic homogeneous form is invariant under the action of the full linear group $G L_{n}(\mathbb{K})$, i.e. if $F$ has a cubic homogeneous form, so has $L \circ F \circ L^{-1}$ for $L \in G L_{n}(\mathbb{K})$.

Let us recall the following important fact. Then

Proposition 3.3 ([BCW, D1). Let $F=I+H$ has a cubic homogeneous form.

$\operatorname{Jac} F=1 \Leftrightarrow$ the matrix $H^{\prime}(x)$ is a nilpotent matrix for every $x \in \mathbb{K}^{n}$.

Proof. Since $\mathbb{K} \subset \mathbb{C}$, we may treat the matrix $H^{\prime}(x)$ as a complex matrix. Note that $H^{\prime}(x)=3 \tilde{H}(x, x, \cdot)$, where $\tilde{H}$ denotes the unique symmetric three-linear mapping such that $\tilde{H}(x, x, x)=H(x)$. Hence $\alpha H^{\prime}(x)=H^{\prime}(\sqrt{\alpha} x)$ for $\alpha \in \mathbb{K}$. Let us write the characteristic equation of the matrix $H^{\prime}(x)$, namely

$$
0=\operatorname{det}\left[\lambda I-H^{\prime}(x)\right]=\lambda^{n} \operatorname{det}\left[I+H^{\prime}(x / \sqrt{-\lambda})\right]=\lambda^{n} \operatorname{Jac} F(x / \sqrt{-\lambda}) .
$$

Assuming that $\operatorname{Jac} F=1$, we get $\lambda^{n}=0$, i.e. all eigenvalues $\lambda$ of the matrix $H^{\prime}(x)$ are equal to 0 . Hence the matrix $H^{\prime}(x)$ is nilpotent.

The converse is obvious as $\operatorname{det}(I+A)=1$ for every nilpotent matrix $A$.

Hence, if $\operatorname{Jac}(I+H)=1$, then by the above proposition the matrix $H_{x}:=$ $\tilde{H}(x, x, \cdot)$ is nilpotent. Therefore for every $x \in \mathbb{K}^{n}$ there exists the index of nilpotency of the matrix $H_{x}$, i.e. a natural number $p(x)$ such that $H_{x}^{p(x)}=0$ and $H_{x}^{p(x)-1} \neq 0$. We define the index of nilpotency of the mapping $F$ to be the number

$$
\text { ind } F:=\sup \left\{p(x) \in \mathbb{N}: H_{x}^{p(x)}=0, H_{x}^{p(x)-1} \neq 0, x \in \mathbb{K}^{n}\right\} .
$$

Obviously $1 \leq p(x) \leq 1+\operatorname{rank} H_{x}$ for every $x \in \mathbb{K}^{n}$. Now we present a theorem which allowed us to reduce the verification of the Generalized Jacobian Conjecture to the investigation of polynomial mappings of the so called cubic linear form.

THEOREM 3.4 ([D3]). In order to verify (GJC) it is sufficient to check it (for every $n>1)$ only for polynomial mappings $F=\left(F_{1}, \ldots, F_{n}\right)$ of the cubic linear form, i.e.

$$
F(x)=\left(x_{1}+\left(a_{1} x\right)^{3}, x_{2}+\left(a_{2} x\right)^{3}, \ldots, x_{n}+\left(a_{n} x\right)^{3}\right),
$$

where $x \in \mathbb{K}^{n}, a_{j}=\left(a_{j}^{1}, \ldots, a_{j}^{n}\right) \in \mathbb{K}^{n}, a_{j} x:=a_{j}^{1} x_{1}+\ldots+a_{j}^{n} x_{n}, j=1, \ldots, n$, having an additional nilpotent property $(\mathrm{N})$ of the matrix $A:=\left[a_{j}^{i}: i, j=1, \ldots, n\right]$, namely

(N) there exists a point $c \in \mathbb{K}^{n}$ such that $A=A_{c}:=F^{\prime}(c)-I$ and ind $A=\operatorname{ind} F$.

Now we recall a theorem which summarizes some partial results on the Generalized Jacobian Conjecture contained in [D1, D3, DR, Wr2]. 
THEOREM 3.5. I. If a polynomial map $F=\left(F_{1}, \ldots, F_{n}\right): \mathbb{K}^{n} \rightarrow \mathbb{K}^{n}$ with Jac $F=1$ has a cubic linear form and if
(i) $\operatorname{rank} A \leq 3$
or
(ii) $\operatorname{corank} A<3$
or
(iii) ind $F=1,2,3, n$,

then $F$ is a polynomial automorphism.

II. If a polynomial map $F=\left(F_{1}, \ldots, F_{n}\right): \mathbb{R}^{n} \rightarrow \mathbb{R}^{n}$ with Jac $F>0$ has a cubic linear form and if $\operatorname{rank} A=1$ or $\operatorname{rank} A=n$, then $F$ is bijective.

For nontrivial applications of the above theorem to the problem of reducibility of certain algebraic sets arising in the Jacobian Problem see [R1].

4. The Jacobian Conjecture in $\mathbb{C}^{2}$. We begin with the following theorem which stresses the connections between the two dimensional Jacobian Conjecture and the problem of the decomposition of $2 \times 2$-matrices over a ring $\mathbf{k}$ as a product of the so called elementary matrices. We call a $2 \times 2$-matrix $A$ over a ring $\mathbf{k}$ elementary if $A$ is of the form

$$
A=\left(\begin{array}{cc}
1 & w \\
0 & 1
\end{array}\right) \quad \text { or } \quad A=\left(\begin{array}{ll}
1 & 0 \\
v & 1
\end{array}\right), \quad \text { where } v, w \in \mathbf{k} .
$$

P. M. Cohn, cf. [Co] has proved that the matrix

$$
\left(\begin{array}{cc}
1+X_{1} X_{2} & X_{1}^{2} \\
-X_{2}^{2} & 1-X_{1} X_{2}
\end{array}\right)
$$

is not elementary. Due to D. Wright, cf. [Wr1], we have the following formulation of the Jacobian Conjecture in $\mathbb{C}^{2}$.

TheOREM 4.1. Let $F=(f, g): \mathbb{C}^{2} \rightarrow \mathbb{C}^{2}$ be a polynomial mapping with Jac $F=1$. The matrix $F^{\prime}\left(X_{1}, X_{2}\right)$ is a product of elementary matrices over the ring $\mathbb{C}\left[X_{1}, X_{2}\right]$ if and only if $F$ is a polynomial automorphism.

Now we present the theorem which uses the analytic approach to the Jacobian Conjecture and combines some results.

TheOREM 4.2. Let $F=(f, g): \mathbb{C}^{2} \rightarrow \mathbb{C}^{2}$ be a polynomial mapping with Jac $F=1$. If , additionally, one of the following conditions is fulfilled:

(i) $g$ has one branch at infinity (i.e. $g$ has one point at $\infty$ and $g$ is analytically irreducible at this point),

(ii) $g-c$ has at most two branches at infinity for an infinite number of $c \in \mathbb{C}$,

(iii) $f$ is proper on $g^{-1}(0)$ (i.e. $\lim |f(x, y)|=\infty$ when $\|(x, y)\| \rightarrow \infty$ and $g(x, y)=0$,

(iv) $\max \left\{\# F^{-1}(w): w \in \mathbb{C}^{2}\right\} \leq 3$,

then $F$ is injective.

Proof. (i) is proved in [A], (ii) in [D2], (iii) in [D2] and, independently, in $[\mathrm{CK}]$. If $F$ is a two sheeted (branched) covering, then injectivity of $F$ was well known, and for three sheeted covering the proof is [Or]. 
Recall that the two dimensional complex Jacobian Conjecture is true if $\max \{\operatorname{deg} f, \operatorname{deg} g\}<100$, cf. [Mo].

5. Global stability problem and the real Jacobian Conjecture. Partial answers to the real Generalized Jacobian Conjecture considered in the cubic linear form was given in Theorem 3.5.II. We think that the remarkable results in the area of the two dimensional real Jacobian Conjecture are due to Meisters and Olech, cf. [MO1, MO2, MO3], and we recall their solution of the two dimensional Global Stability Conjecture for polynomial mappings.

TheOREM 5.1 ([MO2]). Let $F=\left(F_{1}, F_{2}\right)$ be a polynomial map of $\mathbb{R}^{2}, F(0,0)=$ $(0,0)$. Let further $\operatorname{Jac} F\left(x_{1}, x_{2}\right)>0$ and

$$
\operatorname{Tr} F^{\prime}\left(x_{1}, x_{2}\right):=\frac{\partial F_{1}}{\partial x_{1}}\left(x_{1}, x_{2}\right)+\frac{\partial F_{2}}{\partial x_{2}}\left(x_{1}, x_{2}\right)<0
$$

for every $\left(x_{1}, x_{2}\right) \in \mathbb{R}^{2}$. Then the map $F$ is bijective and the autonomous system of differential equations

$$
\dot{x}_{1}(t)=F_{1}\left(x_{1}, x_{2}\right), \dot{x}_{2}(t)=F_{2}\left(x_{1}, x_{2}\right)
$$

is globally asymptotically stable with the rest point $(0,0)$.

We made an attempt to extend the above theorem to the $n$-dimensional case, but we had to impose some additional technical conditions on the eigenvalues of the symmetric part of the matrix of the derivative $F^{\prime}(x)$.

TheOrem $5.2([\mathrm{DT}])$. Let $F=\left(F_{1}, \ldots, F_{n}\right)$ be a polynomial map of $\mathbb{R}^{n}$, $F(0)=0$. Let further $H(y):=\frac{1}{2}\left[F^{\prime}(y)+F^{\prime}(y)^{T}\right]$, let $\lambda_{1}(y), \ldots, \lambda_{n}(y)$ be the eigenvalues of the matrix $H(y)$ and let $\alpha(y):=\max \left\{\left(\lambda_{j}(y)+\lambda_{k}(y)\right): j \neq k\right.$, $j, k=1, \ldots, n\}, y \in \mathbb{R}^{n}$.

Assume that the following conditions hold

(i) $\operatorname{Jac}(y) \neq 0$ for every $y \in \mathbb{R}^{n}$,

(ii) all eigenvalues of the matrix $F^{\prime}(b)$ have negative real parts if $F(b)=0$.

(iii) $\alpha(y) \leq 0$ for every $y \in \mathbb{R}^{n}$.

Then the solution $y(\cdot)=0$ is a GAS solution of the differential equation $(\star)$ $\dot{y}=F(y)$ and the mapping $F$ is bijective.

Meisters and Olech have also obtained interesting results on injectivity of $\mathcal{C}^{1}$ mappings of $\mathbb{R}^{2}$, cf. [MO3], which were improved, namely

TheOrem $5.3([\mathrm{Ch}])$. Let $F=(f, g): \mathbb{R}^{2} \rightarrow \mathbb{R}^{2}$ be a polynomial map and $\pm \mathrm{Jac} F>0$. If there exists a vector $v \in \mathbb{R}^{2}$ and $C>0$ such that

$$
(0,0) \notin \text { convex hull of }\left\{d_{x} F(v): x \in \mathbb{R}^{2},\|x\|>C\right\},
$$

then $F$ is injective. 
As an immediate consequence of the above theorem we have the following:

COROllary 5.4 ([Ch]). If a polynomial map $F=(f, g): \mathbb{R}^{2} \rightarrow \mathbb{R}^{2}$ has the property that Jac $F$ and at least one of the four partial derivatives $\frac{\partial f}{\partial x}, \frac{\partial f}{\partial y}, \frac{\partial g}{\partial x}, \frac{\partial g}{\partial y}$ never vanishes on $\mathbb{R}^{2}$, then $F$ is injective.

\section{References}

[A] S. S. Abhyankar, Local Analytic Geometry, Academic Press, New York, 1964.

[BCW] H. Bass, E. H. Connell and D. Wright, The Jacobian Conjecture: reduction of degree and formal expansion of the inverse, Bull. Amer. Math. Soc. 7 (1982), 287-330.

[BR] A. Białynicki-Birula and M. Rosenlicht, Injective morphisms of real algebraic varieties, Proc. Amer. Math. Soc. 13 (1962), 200-203.

[Ch] Nguyen Van Chau, A sufficient condition for injectivity of polynomial maps on $\mathbb{R}^{2}$, Acta Math. Vietnamica, to appear.

[CK] J. Chạdzyński and T. Krasiński, Properness and the Jacobian Conjecture in $\mathbf{C}^{2}$, Bull. Soc. Sci. Lettres Łódź XIV, 132 (1992), 13-19.

[Co] P. M. Cohn, On the structure of the $G L_{2}$ of a ring, Publ. I.H.E.S. 30 (1966), 365-413.

[D1] L. M. Drużkowski, An effective approach to Keller's Jacobian Conjecture, Math. Ann. 264 (1983), 303-313.

[D2] -, A geometric approach to the Jacobian Conjecture in $\mathbf{C}^{2}$, Ann. Polon. Math. 55 (1991), 95-101.

[D3] - , The Jacobian Conjecture in case of rank or corank less than three, J. Pure Appl Algebra 85 (1993), 233-244.

[D4] -, The Jacobian Conjecture, preprint 492, Institute of Mathematics, Polish Academy of Sciences, Warsaw, 1991.

[DR] L. M. Drużkowski and K. Rusek, The formal inverse and the Jacobian Conjecture Ann. Polon. Math. 46 (1985), 85-90.

[DT] L. M. Drużkowski and H. Tutaj, Differential conditions to verify the Jacobian Conjecture, ibid. 57 (1992), 253-263.

[E] W. Engel, Ein Satz über ganze Cremona Transformationen der Ebene, Math. Ann. 130 (1955), 11-19.

[Es1] A. van den Essen, A criterion to decide if a polynomial map is invertible and to compute the inverse, Comm. Algebra 18 (1990), 3183-3186.

[Es2] - Polynomial maps and the Jacobian Conjecture, Report 9034, Catholic University, Nijmegen, The Netherlands, 1990.

[Es3] - The exotic world of invertible polynomial maps, Nieuw Arch. Wisk. (4) 11 (1993), $21-31$.

[G] W. Gröbner, Sopra un teorema di B. Segre, Atti Accad. Naz. Lincei Rend. Cl. Sci. Fis. Mat. Nat. 31 (1961), 118-122.

[K] O.-H. Keller, Ganze Cremona-Transformationen, Monatshefte Math. Phys. 47 (1939), 299-306.

[KS] T. Krasiński and S. Spodzieja, On linear differential operators related to the $n$ dimensional Jacobian Conjecture, in: Lecture Notes Math. 1524, Springer, 1992, 308-315.

[M] G. H. Meisters, Jacobian problems in differential equations and algebraic geometry, Rocky Mountain J. Math. 12 (1982), 679-705.

[MO1] G. H. Meisters and C. Olech, A poly-flow formulation of the Jacobian Conjecture, Bull. Polish Acad. Sci. 35 (1987), 725-731. 
[MO2] G. H. Meisters and C. Olech, Solution of the Global Asymptotic Stability Jacobian Conjecture for the polynomial case, in: Analyse Mathématique et Applications, Gauthier-Villars, Paris, 1988, 373-381.

[MO3] - - - A Jacobian Condition for injectivity of differentiable plane maps, Ann. Polon. Math. 51 (1990), 249-254.

[Mo] T. T. Moh, On the Jacobian Conjecture and the configuration of roots, J. Reine Angew. Math. 340 (1983), 140-212.

[O] S. Oda, The Jacobian Problem and the simply-connectedness of $\mathbf{A}^{n}$ over a field $\mathbf{k}$ of characteristic zero, preprint, Osaka University, 1980.

[Or] S. Yu. Orevkov, On three-sheeted polynomial mappings of $\mathbf{C}^{2}$, Izv. Akad. Nauk SSSR 50 (6) (1986), 1231-1240 (in Russian).

[P] A. Płoski, On the growth of proper polynomial mappings, Ann. Polon. Math. 45 (1985), 297-309.

[R1] K. Rusek, A geometric approach to Keller's Jacobian Conjecture, Math. Ann. 264 (1983), 315-320.

[R2] -, Polynomial automorphisms, preprint 456, Institute of Mathematics, Polish Academy of Sciences, Warsaw, 1989

[RW] K. Rusek and T. Winiarski, Polynomial automorphisms of $\mathbf{C}^{n}$, Univ. Iagell. Acta Math. 24 (1984), 143-149.

[S1] B. Segre, Corrispondenze di Möbius e Transformazioni cremoniane intere, Atti Accad. Sci. Torino: Cl. Sci. Fis. Mat. Nat. 91 (1956-57), 3-19.

[S2] - Forma differenziali e loro integrali, vol. II, Docet, Roma, 1956.

[S3] - Variazione continua ed omotopia in geometria algebraica, Ann. Mat. Pura Appl. 100 (1960), 149-186.

[St] - On linear differential operators related to the Jacobian Conjecture, J. Pure Appl. Algebra 52 (1989), 175-186.

[V] A. G. Vitushkin, Some examples connected with polynomial mappings in $\mathbb{C}^{n}$, Izv. Akad. Nauk SSSR Ser. Mat. 35 (2) (1971), 269-279.

[W] T. Winiarski, Inverse of polynomial automorphisms of $\mathbb{C}^{n}$, Bull. Acad. Polon. Sci. Math. 27 (1979), 673-674.

[Wr1] D. Wright, The amalgamated free product structure of $G L_{2}\left(k\left[X_{1}, \ldots, X_{n}\right]\right)$ and the weak Jacobian Theorem for two variables, J. Pure Appl. Algebra 12 (1978), 235-251.

[Wr2] D. Wright, The Jacobian Conjecture: linear triangularization for cubics in dimension three, Linear and Multilinear Algebra 34 (1993), 85-97.

[Y] A. V. Yagzhev, On Keller's problem, Sibirsk. Mat. Zh. 21 (1980), 141-150 (in Russian). 